Review began 06/28/2021 Review ended 02/04/2022 Published 02/14/2022 Corrected 03/18/2022

\section{(c) Copyright 2022}

Malik et al. This is an open access article distributed under the terms of the Creative Commons Attribution License CC-BY 4.0., which permits unrestricted use, distribution, and reproduction in any medium, provided the original author and source are credited.

\title{
Corrected: Cognitive Impairment in Type 2 Diabetes Mellitus
}

\author{
Aimen Malik ${ }^{1}$, Mubariz Ahmed ${ }^{2}$, Sarah Mansoor ${ }^{3}$, Saima Ambreen ${ }^{2}$, Basil Usman ${ }^{4}$, Malik Shehryar ${ }^{2}$ \\ 1. Emergency Medicine, Holy Family Hospital, Rawalpindi, PAK 2. Internal Medicine, Holy Family Hospital, Rawalpindi, \\ PAK 3. Internal Medicine, Diana, Princess of Wales Hospital, Grimsby, GBR 4. Medical School, Rawalpindi Medical \\ University, Rawalpindi, PAK
}

Corresponding author: Mubariz Ahmed, mubarizahmed27@gmail.com

\section{This article has been corrected.}

Correction date: March 18, 2022. Cite this correction as Malik A, Ahmed M, Mansoor S, et al. (March 18, 2022) Correction: Cognitive Impairment in Type 2 Diabetes Mellitus. Cureus 14(3): c59. doi:10.7759/cureus.c59.

This article has been corrected to resolve an issue regarding attribution of the licensed tool used in data collection. The authors started data collection in June 2019 with an Urdu translation of Mini Mental State Examination (MMSE) that was commonly used and in circulation in Pakistan for judging cognitive status of patients. Unfortunately at that time the authors did not know that the MMSE had been licensed, and that they would have to purchase authorized copies from the copyright holder Psychological Assessment Resources, Inc for use in their study. This issue was brought to their attention prior to publication and the authors then contacted the license holding company for retroactive permission to publish and paid for the rights.

Per the permission agreement, the authors were required to state in the publication that unauthorized Urdu MMSE copies were used. This was added to the Intellectual Property disclosures section with the authors believing they had satisfied this condition. However, after publication, Psychological Assessment Resources required the following corrections, which have since been made:

- The following statement was moved from the Disclosures section to the second paragraph of the Materials and Methods section: "The authors without prior knowledge that the tool had been licensed used unauthorized copies of Urdu MMSE for data collection. Permission for publication of data obtained from administering MMSE to 332 patients was retroactively obtained from Psychological Assessment Resources, Inc."

- Since the copies of Urdu MMSE were unauthorized, they cannot be validated and the reference to validation should be removed. As a result, the following sentence and accompanying reference have been removed and the remaining in-text citations renumbered to ensure accuracy with the revised reference list: "The Urdu translation of MMSE has previously been validated, and a cutoff value of 24 was found to maximize sensitivity and specificity (69\% and $93 \%$, respectively) when subjects of all education levels were collectively considered [16]." [16] Awan S, Shahbaz N, Akhtar SW, et al.: Validation study of the Mini-Mental State Examination in Urdu language for Pakistani population. Open Neurol J. 2015, 9:53-8. 10.2174/1874205X01509010053

\section{Abstract \\ Objective}

To determine the incidence of cognitive impairment established on the mini-mental state assessment in type 2 diabetic patients presenting at Holy Family Hospital, Rawalpindi.

\section{Materials and methods}

This cross-sectional descriptive study was carried out from June 2019 to December 2019. Individuals with a diagnosis of type 2 diabetes mellitus were included, and detailed history, physical examination, and biochemical variables were noted. They were assessed through Mini-Mental State Examination (MMSE) (Urdu translation) to look for the primary outcome variable, i.e., cognitive impairment. All patients with type 2 diabetes mellitus diagnosed at least one year back, irrespective of gender, were included in this 
investigation. Patients with a previous history of head injury, epilepsy, stroke, those on an antidepressant or antipsychotic medications, those with deranged renal function tests, and those already diagnosed with dementia were excluded from the study.

\section{Results}

Three hundred thirty-two patients meeting the inclusion criteria were included in the study. The mean \pm standard deviation age of the study population was $65.32 \pm 11.33$ years, with maximum age being 80 years and the minimum being 50 years. Two hundred patients (60.24\%) were below 65 years of age, and 132 patients (39.76\%) were 65 years of age or above. Two hundred sixteen (65.06\%) were males, and 116 (34.96\%) were females. The mean duration of diabetes mellitus (DM) was $10.17 \pm 4.81$. The mean MMSE score was $22.69 \pm 5.26$. Out of 332 patients, 81 (24.4\%) patients had cognitive impairment. Patients who were 65 or older had a significantly higher proportion of cognitive impairment, compared to those below 65 years of age $(p$-value $=0.0214)$. There was no significant difference in the proportion of cognitively impaired patients between males and females $(\mathrm{p}$-value $=0.2497$ ). Similarly, there was no significant difference between those who were diagnosed with type 2 diabetes for 10 years or more and those who were diagnosed less than 10 years ago $(\mathrm{p}$-value $=0.3791)$.

\section{Conclusion}

Cognitive impairment is common in individuals having type 2 diabetes mellitus. It is also associated with the increasing age of diabetic patients. However, cognitive impairment in type 2 diabetes mellitus is not associated with gender. In addition, there is no significant difference in cognitive impairment between the patients who were diagnosed with diabetes more than 10 years ago and those who had it diagnosed less than 10 years ago.

Categories: Endocrinology/Diabetes/Metabolism, Internal Medicine, Neurology Keywords: cognitive, brain, diabetes, encephalopathy, mmse

\section{Introduction}

Diabetes mellitus (DM) is a chronic disorder of elevated blood glucose. It is classified into two distinct types (type 1 and 2). Type 1 diabetes mellitus is characterized by autoimmune destruction of beta cells in the pancreas leading to absolute insulin deficiency. Type 2 diabetes mellitus is characterized by different combinations of insulin resistance and insulin deficiency. As per WHO, diabetes is diagnosed with one or a combination of the following criteria: a fasting plasma glucose concentration equivalent to or greater than $7.0 \mathrm{mmol} /$ liter $(126 \mathrm{mg} / \mathrm{dL})$, two hours plasma glucose concentration equivalent to or greater than 11.1 $\mathrm{mmol} /$ liter $(200 \mathrm{mg} / \mathrm{dL}$ ) during a 75 -g oral glucose tolerance test, and/or hemoglobin A1C (HbA1c) equivalent to or beyond $6.5 \%(48 \mathrm{mmol} / \mathrm{mol})[1]$.

Diabetes occurs in 5\%-10\% of the population. Majority of the affected population (95\%) has type 2 diabetes mellitus. It is estimated 463 million people were affected with diabetes in 2019 [2]. Over the past 25 years, the prevalence of diabetes has doubled in men and increased by $60 \%$ in women [3]. In Pakistan, the overall weighted prevalence of diabetes was recorded up to $26.3 \%$ [4].

Various micro- and macrovascular complications of diabetes mean that the disease is a major factor of morbidity and mortality worldwide. These include cardiovascular and cerebrovascular disease, peripheral vascular disease, retinopathy, and end-stage renal disease [5]. Diabetes multiplies the risk of all-site cancer (except prostatic carcinoma) [5]. It also increases the risk of mental health illnesses among patients, such as depression [6]. These complications are generally well known to the healthcare providers and are usually dealt with adequately, according to the resources available.

However, a problem commonly ignored by healthcare providers is the link between diabetes and cognitive dysfunction. This is despite the relationship being well established in the literature. In recent years, several studies have shown strong evidence for the presence of cognitive impairment in diabetes [7-9]. A recent systematic review and meta-analysis estimated the prevalence of mild cognitive impairment type 2 diabetes mellitus (T2DM) patients to be as high as $45 \%$ [10].

Insulin resistance and obesity (which are shared risk factors for both diabetes and cognitive impairment), chronic low-grade inflammation and hyperglycemia or hypoglycemia, and cardiovascular complications of diabetes may be some of the risk factors implicated in development of cognitive impairment in patients $[11,12]$. Diabetes is known to adversely affect several cognitive domains. These include memory and processing speed, although executive function may be spared $[13,14]$.

Since there is a dearth of studies conducted in our region exploring the relationship between type 2 diabetes and cognitive impairment, we wanted to conduct this investigation. Knowledge of this prevalence would help healthcare providers give it due consideration while managing the complications of diabetes. 


\section{Cureus}

\section{Materials And Methods}

This descriptive cross-sectional study was conducted at Medicine Unit I, Holy Family Hospital, Rawalpindi, Pakistan, from June 2019 to December 2019. Ethical approval was taken from the institutional research forum of the Rawalpindi Medical University. Patients with age greater than 50 years, random blood sugar greater than $200 \mathrm{mg} / \mathrm{dL}$, fasting blood sugar level greater than $126 \mathrm{mg} / \mathrm{dL}$, or HbA1c levels greater than $6.5 \%$ were defined as having type 2 diabetes mellitus. All patients with type 2 diabetes mellitus diagnosed at least one year back, irrespective of gender, were included in the study. Patients with a history of head injury, epilepsy, stroke, those on an antidepressant or antipsychotic medication, those with deranged renal function tests, and those already diagnosed with dementia were excluded from the study. Three hundred thirty-two patients were selected using consecutive (nonprobability) sampling. All the patients were explained about the nature and purpose of the study. Informed written consent for participation was obtained. Patients' sociodemographic details including years of education were recorded in a proforma.

An Urdu translation of Mini-Mental State Examination (MMSE) was applied through the interview from each patient to assess cognitive impairment. The MMSE takes into account various domains of cognitive functioning. These include spatial and temporal orientation, immediate memory, attention/concentration, delayed recall, and language [15]. Patients with scores 23 or below out of 30 were considered cognitively impaired. The authors, without prior knowledge that the tool had been licensed, used unauthorized copies of Urdu MMSE for data collection. Permission for publication of data obtained from administering MMSE to 332 patients was retroactively obtained from Psychological Assessment Resources, Inc.

The collected data were analyzed using the statistical software SPSS-17 (IBM, New York, United States). For categorical variables like gender and presence or absence of cognitive impairments, frequencies along with percentages were calculated. For quantitative variables like age and the exact MMSE score, the mean and standard deviation was calculated. Factors such as age, gender, and duration of diabetes were controlled through stratification. Chi-square test was applied poststratification. P-value less than 0.05 was considered significant.

\section{Results}

The mean \pm standard deviation age of the study population was $65.32 \pm 11.33$ years, with the maximum age being 80 years and the minimum being 50 years. Two hundred patients (60.24\%) were below 65 years of age, and 132 patients (39.76\%) were 65 years of age or above (Figure 1). Two hundred sixteen (65.06\%) were males, and 116 (34.96\%) were females (Figure 2). The mean duration of DM was $10.17 \pm 4.81$. The mean MMSE score was $22.69 \pm 5.26$

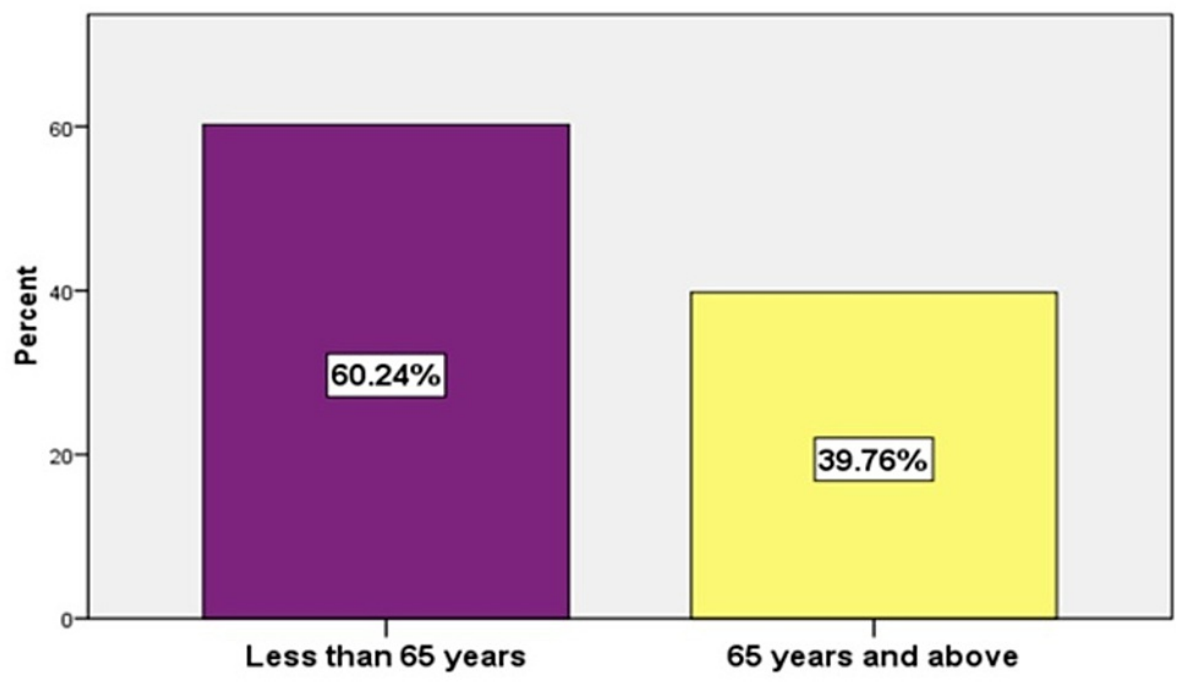

FIGURE 1: Distribution of patients by age 


\section{Cureus}

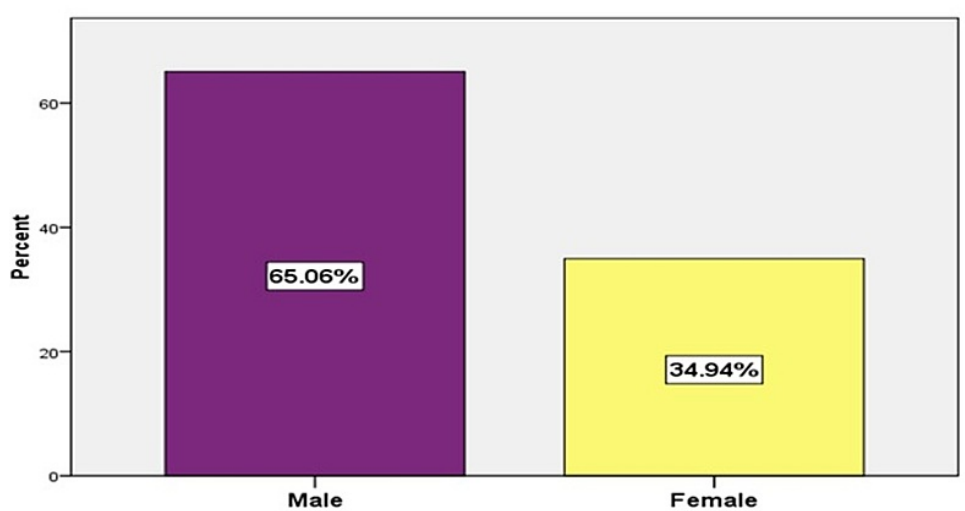

Figure 2

\section{FIGURE 2: Distribution of patients by gender}

Out of 332 patients, 81 (24.4\%) patients had cognitive impairment (Figure 3). Patients who were 65 or older had a significantly higher proportion of cognitive impairment, compared to those below 65 years of age (pvalue $=0.0214)($ Table 1$)$. There was no significant difference in the proportion of cognitively impaired patients between males and females (p-value $=0.2497)($ Table 1$)$. Similarly, there was no significant difference in cognitive impairment between those who had type 2 diabetes mellitus for 10 years or more and those who had type 2 diabetes mellitus for less than 10 years $(\mathrm{p}$-value $=0.3791)($ Table 1$)$.

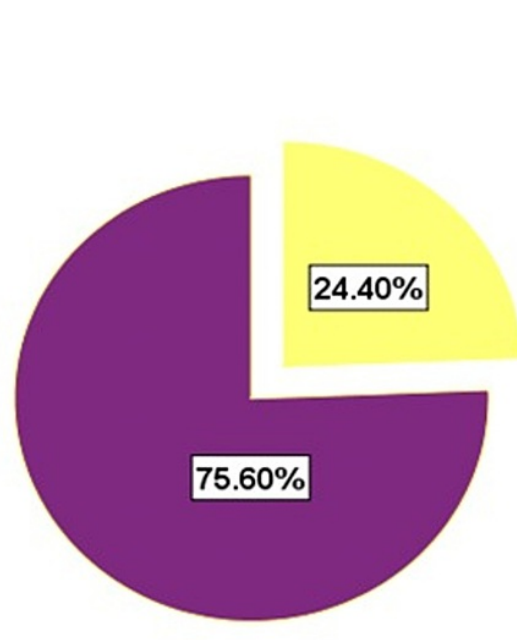

\section{Figure 3}

FIGURE 3: Proportion of cognitively impaired patients versus those not cognitively impaired 


\section{Cureus}

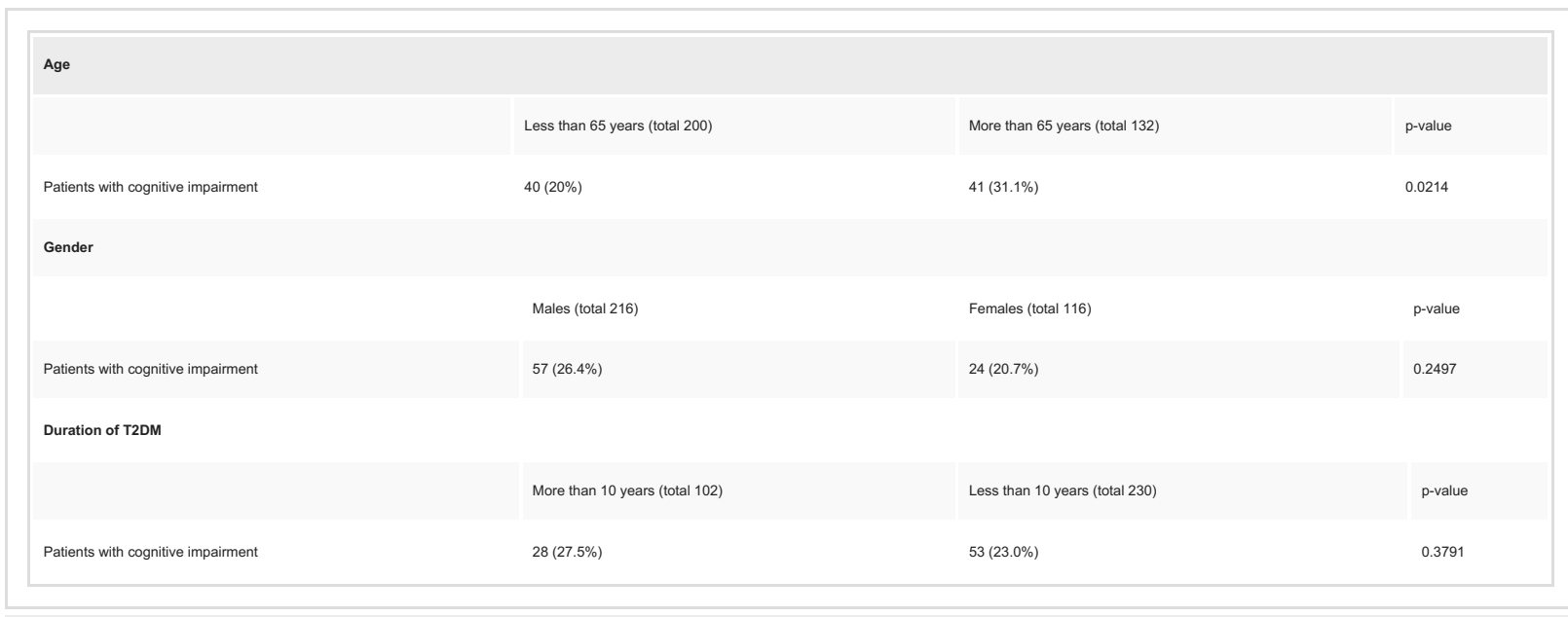

TABLE 1: Analysis of cognitive impairment with age, gender, and duration of type 2 diabetes mellitus

T2DM: type 2 diabetes mellitus.

\section{Discussion}

The results of our study ascertain that almost one in four of the patients suffering from type 2 diabetes mellitus also suffers from cognitive impairment; therefore, cognitive dysfunction is not uncommon in patients with T2DM. This proportion is in line with the findings in the literature. Khullar et al. found this proportion to be $33.73 \%$ [16]. Other studies have shown it to range from $3 \%$ to $23 \%[17,18]$.

We want to emphasize that cognitive dysfunction in type 2 diabetes mellitus is almost as common as its other micro- and macrovascular complications. Awareness of this important complication can help in better counseling of the patients and their families. It can lead to directing more material resources and expertise toward the issue. It can also lead to a better multidisciplinary approach, such as the involvement of the neurologist for better patient management.

Cognitive impairment might be one of the multitudes of reasons for poor compliance of the patients to lifestyle modifications and pharmacotherapy used to manage the disease. Patient education regarding cognitive impairment would help them better understand the problems they are facing. This might lead to better compliance to treatment and, as a result, good glycemic control. Also, education of the family and other caregivers regarding the presence of cognitive dysfunction might help them better understand the problems the patient is facing, and as a result, patient care would improve. In summary, awareness of cognitive dysfunction in type 2 diabetics among physicians, patients, and their caregivers can go a long way in improving patients' quality of life.

In addition to this, our results show that increasing age is a risk factor for the development of cognitive impairment in type 2 diabetics. This relationship is also well established in the literature [19]. Since diabetes mellitus [20] and old age are both independent risk factors for developing Alzheimer's disease, vascular dementia, and other disorders resulting in the culmination of cognitive decline, a combination of both can contribute to a higher incidence of cognitive impairment among older diabetics.

The present study showed no significant difference in cognitive impairment between the genders. This is in contrast to other studies, which have described that the diabetic women have double the risk of neurocognitive impairment compared to men, and female gender is an independent risk factor for development of neurocognitive deficit [21,22]. One study even described a 3.75 times risk of cognitive impairment in women compared to men [23]. Perhaps, a larger sample size could have shed more light on this relationship.

The present study also showed no significant relationship between the duration of T2DM with cognitive impairment. This is also contrary to many previous studies investigating the association. One study showed that patients who have been diagnosed with T2DM for five years or more perform worse in aspects of cognition such as logical memory and word fluency compared to those who have been diagnosed newly [24]. A study of the Iranian population reported a negative correlation between MMSE scores and the duration of T2DM [25]. Similarly, a study conducted in India showed that diabetic individuals with a duration of diabetes for more than 10 years were 4.34 times more likely to develop cognitive impairment compared to the newly diagnosed diabetics [26]. We propose that more studies should be conducted in our region, so this 
relationship could be explored further.

Our results might have been influenced by certain potential limitations. Firstly, age and education can influence MMSE scoring to judge neurocognition. Secondly, since the sensitivity of MMSE for timed elements of executive functions and memory is low, residual confounding for them may have remained to be exposed. Thirdly, the number of diabetic patients included in the study might be small and a larger cohort could have better elucidated the relationships we investigated.

\section{Conclusions}

Cognitive impairment is common in individuals having type 2 diabetes mellitus. It is also associated with the increasing age of diabetic patients. However, cognitive impairment in type 2 diabetes mellitus is not associated with gender. In addition, there is no significant difference in cognitive impairment between the patients who were diagnosed with diabetes more than 10 years ago and those who had it diagnosed less than 10 years ago.

\section{Additional Information \\ Disclosures}

Human subjects: Consent was obtained or waived by all participants in this study. Institutional Research Forum RMC and Allied Hospitals issued approval R-07/RMC/16. The research proposal titled as "COGNITIVE IMPAIRMENT IN TYPE 2 DIABETIC PATIENTS PRESENTING AT HOLY FAMILY HOSPITAL RAWALPINDI," submitted by Dr. Aimen Malik, Post Graduate Trainee at Department of Medicine, Holy Family Hospital Rawalpindi, conforms to the accepted ethical standards established by Institution Research Forum of Rawalpindi Medical University (RMC). Therefore, her research proposal has been approved by Institutional Research Forum and The Research and Ethical Committee of RMC, and she is allowed to initiate her research at RMC. Animal subjects: All authors have confirmed that this study did not involve animal subjects or tissue. Conflicts of interest: In compliance with the ICMJE uniform disclosure form, all authors declare the following: Payment/services info: All authors have declared that no financial support was received from any organization for the submitted work. Financial relationships: All authors have declared that they have no financial relationships at present or within the previous three years with any organizations that might have an interest in the submitted work. Other relationships: All authors have declared that there are no other relationships or activities that could appear to have influenced the submitted work.

\section{References}

1. WHO: Definition and diagnosis of diabetes mellitus and intermediate hyperglycemia: report of a WHO/IDF consultation. Geneva: World Health Organization. (2006). Accessed: December 24, 2020: https://www.who.int/diabetes/publications/Definition\%20and\%20diagnosis\%20of\%20diabetes_new.pdf.

2. Saeedi P, Salpea P, Karuranga S, et al.: Mortality attributable to diabetes in 20-79 years old adults, 2019 estimates: results from the International Diabetes Federation Diabetes Atlas, 9th edition. Diabetes Res Clin Pract. 2020, 162:108086. 10.1016/j.diabres.2020.108086

3. Worldwide trends in diabetes since 1980: a pooled analysis of 751 population-based studies with 4.4 million participants. Lancet. 2016, 387:1513-30. 10.1016/S0140-6736(16)00618-8

4. Basit A, Fawwad A, Qureshi H, Shera AS: Prevalence of diabetes, pre-diabetes and associated risk factors: second National Diabetes Survey of Pakistan (NDSP), 2016-2017. BMJ Open. 2018, 8:e020961. 10.1136/bmjopen-2017-020961

5. Chan JC, Lim L-L, Wareham NJ, et al.: The Lancet Commission on diabetes: using data to transform diabetes care and patient lives. Lancet. 2020, 396:2019-82. 10.1016/S0140-6736(20)32374-6

6. Pan A, Lucas M, Sun Q, et al.: Bidirectional association between depression and type 2 diabetes mellitus in women. Arch Intern Med. 2010, 170:1884-91. 10.1001/archinternmed.2010.356

7. Pasquier F: Diabetes and cognitive impairment: how to evaluate the cognitive status? . Diabetes Metab. 2010, 36:100-5. 10.1016/S1262-3636(10)70475-4

8. Paul KC, Jerrett M, Ritz B: Type 2 diabetes mellitus and Alzheimer's disease: overlapping biologic mechanisms and environmental risk factors. Curr Environ Health Rep. 2018, 5:44-58. 10.1007/s40572-0180176-1

9. Umegaki H: Type 2 diabetes as a risk factor for cognitive impairment: current insights . Clin Interv Aging. 2014, 2014:1011-9. 10.2147/CIA.S48926

10. You Y, Liu Z, Chen Y, et al.: The prevalence of mild cognitive impairment in type 2 diabetes mellitus patients: a systematic review and meta-analysis. Acta Diabetol. 2021, 58:671-85. 10.1007/s00592-02001648-9

11. Middleton LE, Yaffe K: Promising strategies for the prevention of dementia . Arch Neurol. 2009, 66:1210-5. 10.1001/archneurol.2009.201

12. Kalyani RR, Corriere M, Ferrucci L: Age-related and disease-related muscle loss: the effect of diabetes, obesity, and other diseases. Lancet Diabetes Endocrinol. 2014, 2:819-29. 10.1016/S2213-8587(14)70034-8

13. Awad N, Gagnon M, Messier C: The relationship between impaired glucose tolerance, type 2 diabetes, and cognitive function. J Clin Exp Neuropsychol. 2004, 26:1044-80. 10.1080/13803390490514875

14. Saczynski JS, Jónsdóttir MK, Garcia ME, et al.: Cognitive impairment: an increasingly important complication of type 2 diabetes: the age, gene/environment susceptibility: Reykjavik study. Am J Epidemiol. 2008, 168:1132-9. 10.1093/aje/kwn228

15. Folstein MF, Folstein SE, McHugh PR: "Mini-mental state”: a practical method for grading the cognitive 
state of patients for the clinician. J Psychiatr Res. 1975, 12:189-98. 10.1016/0022-3956(75)90026-6

16. Khullar S, Dhillon H, Kaur G, et al.: The prevalence and predictors of depression in type 2 diabetic population of Punjab. Community Ment Health J. 2016, 52:479-83. 10.1007/s10597-015-9985-y

17. Allen KV, Frier BM, Strachan MW: The relationship between type 2 diabetes and cognitive dysfunction: longitudinal studies and their methodological limitations. Eur J Pharmacol. 2004, 490:169-75. 10.1016/j.ejphar.2004.02.054

18. Ryan CM, Freed MI, Rood JA, Cobitz AR, Waterhouse BR, Strachan MW: Improving metabolic control leads to better working memory in adults with type 2 diabetes. Diabetes Care. 2006, 29:345-51. 10.2337/diacare.29.02.06.dc05-1626

19. Bruce DG, Casey GP, Grange V, et al.: Cognitive impairment, physical disability and depressive symptoms in older diabetic patients: the Fremantle Cognition in Diabetes Study. Diabetes Res Clin Pract. 2003, 61:59-67. 10.1016/S0168-8227(03)00084-6

20. Zilliox LA, Chadrasekaran K, Kwan JY, Russell JW: Diabetes and cognitive impairment. Curr Diab Rep. 2016, 16:87. 10.1007/s11892-016-0775-X

21. Ho SC, Woo J, Sham A, Chan SG, Yu AL: A 3-year follow-up study of social, lifestyle and health predictors of cognitive impairment in a Chinese older cohort. Int J Epidemiol. 2001, 30:1389-96. 10.1093/ije/30.6.1389

22. Irvine K, Laws KR, Gale TM, Kondel TK: Greater cognitive deterioration in women than men with Alzheimer's disease: a meta analysis. J Clin Exp Neuropsychol. 2012, 34:989-98. 10.1080/13803395.2012.712676

23. Yu ES, Liu WT, Levy P, et al.: Cognitive impairment among elderly adults in Shanghai, China . J Gerontol. 1989, 44:S97-106. 10.1093/geronj/44.3.s97

24. Elias PK, Elias MF, D'Agostino RB, Cupples LA, Wilson PW, Silbershatz H, Wolf PA: NIDDM and blood pressure as risk factors for poor cognitive performance: the Framingham Study. Diabetes Care. 1997, 20:1388-95. 10.2337/diacare.20.9.1388

25. Gottesman RF, Rawlings AM, Sharrett AR, et al.: Impact of differential attrition on the association of education with cognitive change over 20 years of follow-up: the ARIC neurocognitive study. Am J Epidemiol. 2014, 179:956-66. 10.1093/aje/kwu020

26. Abbott MA, Wells DG, Fallon JR: The insulin receptor tyrosine kinase substrate $\mathrm{p} 58 / 53$ and the insulin receptor are components of CNS synapses. J Neurosci. 1999, 19:7300-8. 10.1523/JNEUROSCI.19-1707300.1999 